\title{
Long-Term Trends and Its Best Functional Form Estimation of Yearly Maximum and Minimum Temperatures at Cotonou City by Improved Complete Ensemble Empirical Mode Decomposition with Adaptive Noise Method
}

\author{
Médard Noukpo Agbazo ${ }^{1,2 *}$, Joseph Adébiyi Adéchinan³, Gabin Koto N'gobi², Joseph Bessou ${ }^{4}$ \\ ${ }^{1}$ International Chair in Mathematical Physics and Applications (CIPMA-Chair Unesco), Université d'Abomey-Calavi, UAC, \\ Cotonou, Bénin \\ ${ }^{2}$ Laboratoire de Physique du Rayonnement (LPR), Faculté des Sciences et Techniques, Université d'Abomey-Calavi, UAC, \\ Cotonou, Bénin \\ ${ }^{3}$ Département de Physique, Faculté des Sciences et Techniques, Université Nationale des Sciences, Technologies, Ingénierie et \\ Mathématiques (UNSTIM), Abomey, Bénin \\ ${ }^{4}$ Agence Nationale de la Météorologie du Bénin, Meteo, Bénin \\ Email: ^agbmednou@gmail.com, ^agbazomedard@yahoo.fr
}

How to cite this paper: Agbazo, M.N., Adéchinan, J.A., Koto N'gobi, G. and Bessou, J. (2022) Long-Term Trends and Its Best Functional Form Estimation of Yearly Maximum and Minimum Temperatures at Cotonou City by Improved Complete Ensemble Empirical Mode Decomposition with Adaptive Noise Method. Atmospheric and Climate Sciences, 12, 31-42. https://doi.org/10.4236/acs.2022.121003

Received: September 24, 2021 Accepted: November 23, 2021 Published: November 26, 2021

Copyright ( 2022 by author(s) and Scientific Research Publishing Inc. This work is licensed under the Creative Commons Attribution International License (CC BY 4.0).

http://creativecommons.org/licenses/by/4.0/ (c) (i) Open Access

\begin{abstract}
The understanding of the long-term trend in climatic variables is necessary for the climate change impacts studies and for modeling several processes in environmental engineering. However, for climatic variables, long-term trend is usually unknown whether there is a trend component and, if so, the functional form of this trend is also unknown. In this context, a conventional strategy consists to assume randomly the shape of the local trends in the time series. For example, the polynomial forms with random order are arbitrarily chosen as the shape of the trend without any previous justification. This study aims to 1) estimate the real long-term nonlinear trend and the changing rate of the yearly high temperature among the daily minimum (YHTaDMinT) and maximum temperatures (YHTaDMaxT) observed at Cotonou city, 2) find out for these real trend and trend increment, the best polynomial trend model among four trend models (linear, quadratic, third-order and fourthorder polynomial function). For both time series, the results show that $\mathrm{YH}$ TaDMinT and YHTaDMaxT time series are characterized by nonlinear and monotonically increasing trend. The trend increments present different phases in their nonmonotone variations. Among the four trend estimations models, the trend obtained by third-order and fourth-order polynomial functions ex-
\end{abstract}


hibits a close pattern with the real long-term nonlinear trend given by the Improved Complete Ensemble Empirical Mode Decomposition with Adaptive Noise (ICEEMDAN). But, the fourth-order polynomial function is optimal, therefore, it can be used as the functional form of trend. In the trend increment case, for the YHTaDMaxT time series, the fourth-order fit is systematically the best among the four proposed trend models. Whereas for the YHTaDMinT time series, the third-order and fourth-order polynomial functions present the same performance. They can both be used as the functional form of trend increments. Overall, the fourth-order polynomial function presents a good performance in terms of trend and trend increments estimation.

\section{Keywords}

Long-Term Trends, Polynomial Trend Models, Trend Increment, ICEEMDAN, Extrema Temperature

\section{Introduction}

To modelize several processes in environmental engineering, agriculture, climatology and hydrology, the understanding of the real nonlinear trend and trend model's estimation of climate variables as extrema temperatures are necessary [1]. Moreover, climate change impacts are evaluated through trend analysis of climate variables [2]. Climate change is marked with variation in temperature, which can directly influence the hydrological processes [3] [4] [5]. In Benin Republic, a sub-Saharan country affected by climate change effects, few studies are focused on the real nonlinear trend investigation and on the estimation of the functional form of this trend in the extrema temperatures during historical period. In this country, one of the major challenges for hydrologists and meteorologists is the estimation of the real functional form of historical climate variables trend. Indeed, if there is a trend in a real-time series of historical climatic variables, the functional form of this trend is usually unknown. In this context, certain authors assume randomly the form of the local trends as a polynomial function with arbitrarily chosen order (Abaje and Oladipo [6], Gadedjisso-Tossou et al. [7], Agbazo et al. [8]). Generally, linear regression technique is used to analyze the trends in the climatic variables time series. This situation can lead to erroneous conclusions.

Nowadays, to overcome these difficulties and to correctly represent the trend in climatic variables, with higher accuracy without ignorance of its nonstationarity and nonlinearity characteristics, some improved methods are developed in literature. Among these methods one can cite, the Empirical Mode Decomposition (EMD) developed by Huang et al. [9], and its variations, such as the Ensemble Empirical Mode Decomposition (EEMD) developed by Wu and Huang [10], Complete Ensemble Empirical Mode Decomposition with Adaptive Noise (CEEMDAN) developed by Torres et al. [11] and Improve Complete Ensemble 
Empirical Mode Decomposition with Adaptive Noise (ICEEMDAN) developed by Colominas et al. [12]. The CEEMDAN is an important improvement of EEMD and ICEEMDAN is a further improvement of CEEMDAN [11] [12].

Based on ICEEMDAN method, the purpose of this article is, firstly to estimate the real long-term nonlinear trend and the changing rate of the yearly high temperature among the daily minimum (YHTaDMinT) and maximum temperatures (YHTaDMaxT) observed at Cotonou city, the most industrialized and developed city in Benin Republic, secondly, to find out the best (optimal) polynomial forms of these nonlinear trends and trend increments. The article is structured as follows: Section 2 describes data sets and the methods involved. The results are presented and interpreted in Section 3. Finally, a summary with concluding remarks forms and outlook for further research ends the study in Section 4.

\section{Materials and Methods}

\subsection{Materials}

Cotonou is located in the southern part of Benin Republic (West Africa), closer to the Atlantic Ocean. It is characterized by four seasons each year: a long rainy season (April to July) followed by a short dry season (August to September) and a short rainy season (October to November) followed by long dry season (December to March). In this study, the daily maximum temperature and minimum temperature data are recorded from 1953 to 2019 at Cotonou Airport's synoptic station and provided by "Météo-Benin". From these data, one generates the yearly high temperature among the daily minimum (YHTaDMinT) and the yearly high temperature among the daily maximum temperatures (YHTaDMaxT) observed during each year. Figure 1 shows that YHTaDMinT and YHTaDMaxT time series vary regarding to the year. The minimum values of $\mathrm{YH}-$ TaDMinT and YHTaDMaxT are respectively greater than $27^{\circ} \mathrm{C}$ and $32^{\circ} \mathrm{C}$.

\subsection{Methods}

Improved Complete Ensemble Empirical Mode Decomposition with adaptive noise, namely ICEEMDAN, developed by Colominas et al. [9] is used for estimating nonlinear trends and trend increments in YHTaDMinT and YHTaDMaxT time series. The ICEEMDAN is an important improved version of the complete ensemble empirical mode decomposition with adaptive noise, CEEMDAN (developed by Torres et al. [11]). It is an expansion algorithm of Empirical Mode Decomposition (EMD), which is an adaptive (data-driven) method developed by Huang et al. [9] to analyze nonstationary time series stemming from nonlinear systems. According to Colominas et al. [12], ICEEMDAN's main steps can be described as follows:

1) Construct the signal $x^{(i)}(n)$ which is obtained after the specific white noise is added into the original signal $x(n)$ :

$$
x^{(i)}(n)=x(n)+\alpha_{0} E_{1}\left(\mu^{(i)}(n)\right)
$$




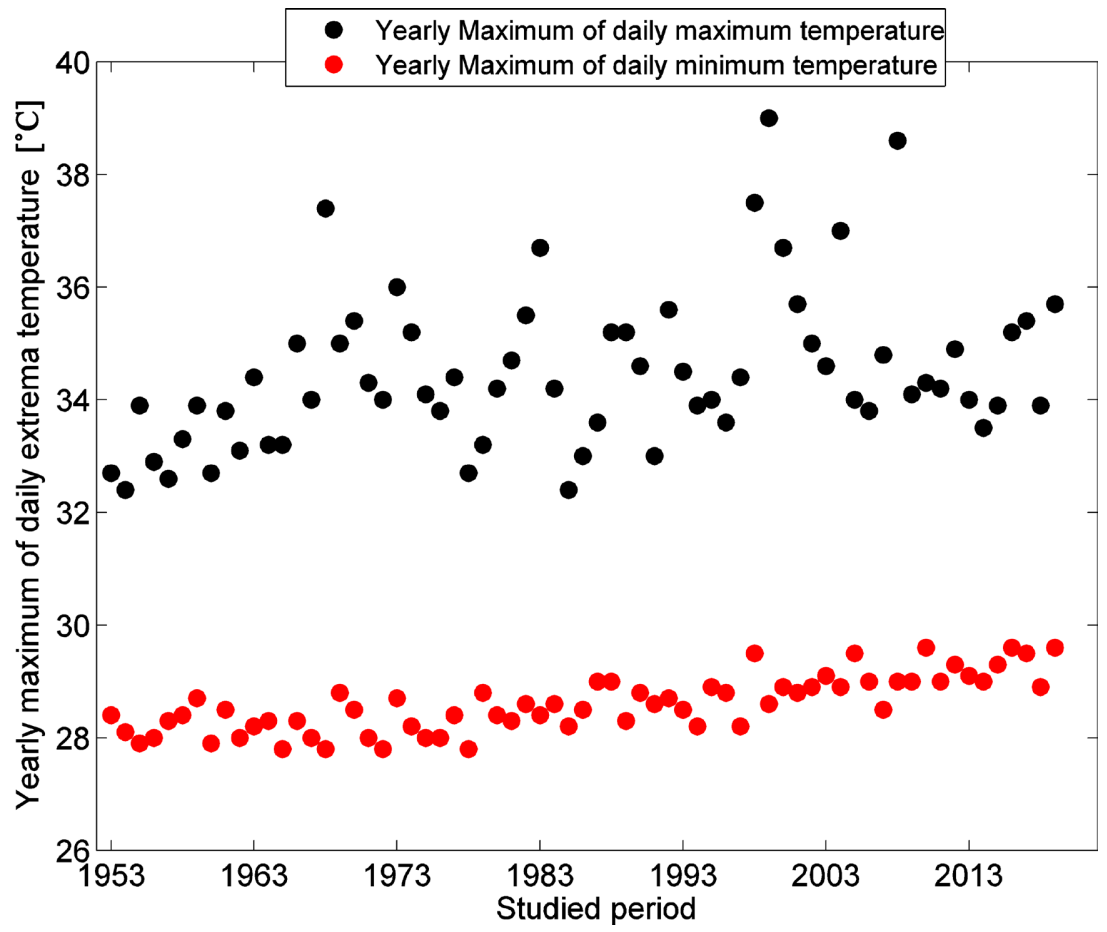

Figure 1. Temporal variation of YHTaDMinT and YHTaDMaxT temperature. YHTaDMinT and YHTaDMaxT time series are respectively in red and black.

$\alpha_{0}$ is the level of noise; $i$ is the added times of noise; $i=1,2, \cdots, N ; \mu^{(i)}(n)$ is the noise with zero-mean and unit variance; and $E_{1}\left(\mu^{(i)}(n)\right)$ is the first EMD component of the white noise $\mu^{(i)}(n)$.

2) Decompose the $\mathrm{N}$ signals by EMD method to obtain the first intrinsic mode function (IMF) component of ICEEMDAN as:

$$
\operatorname{imf}_{1}(n)=x(n)-\left\langle M\left(x^{(i)}(n)\right)\right\rangle
$$

where $\langle$.$\rangle represents the mean value, M($.$) is the operation which produces$ the local average of the envelope that satisfies the IMF sifting threshold and $\operatorname{imf}_{1}$ is the first IMF.

3) Construct the second signal $\left\langle M\left(x^{(i)}(n)\right)\right\rangle+\alpha_{1} E_{2}\left(\mu^{(i)}(n)\right)$. This signal is decomposed by EMD and one obtains the second IMF component of ICEEMDAN:

$$
\operatorname{imf}_{2}(n)=r_{1}(n)-r_{2}(n)=r_{1}(n)-\left\langle M\left(r_{1}(n)+\alpha_{1} E_{2}\left(\mu^{(i)}(n)\right)\right)\right\rangle
$$

where $r_{1}(n)=\left\langle M\left(x^{(i)}(n)\right)\right\rangle$ is the residual obtained after the first decomposition.

4) Construct the $k$ th signal $r_{k-1}(n)+\alpha_{k-1} E_{k}\left(\mu^{(i)}(n)\right)$. This signal is decomposed by EMD and obtains the $k$ th IMF component of ICEEMDAN:

$$
\operatorname{imf}_{k}(n)=r_{k-1}(n)-r_{k}(n)=r_{k-1}(n)-\left\langle M\left(r_{k}(n)+\alpha_{k-1} E_{k}\left(\mu^{(i)}(n)\right)\right)\right\rangle
$$

$r_{k}(n)$ is the further residual after the $k$ th decomposition, and $k=1,2, \cdots, K$.

5) Repeat step 4 for the next $k$ until the residual cannot be decomposed any further, the residual is monotonous, or its amplitude is less than the indicated 
threshold. Then, the decomposition stops.

To evaluate the sensitivity of the nonlinear trend obtained by ICEEMDAN to the $\alpha_{k}$ values, the ICEEMDAN algorithm has been implemented for different values of $\alpha_{k}$. Colominas et al. [12] have recommended $\alpha_{k}$ values between 0.1 and 0.3. In this study, $\alpha_{k}$ values vary from 0.1 to 0.5 with step of 0.1 to evaluate the effect of large interval.

In order to find out the best (optimal) functional form of the real long-term nonlinear trend and its changing rate, different polynomials functions (linear, quadratic, third-order and fourth-order) are used to modelize this trend. Four main indicators are used to clearly reflect the error of each polynomial function's result and evaluate the fitting accuracy: the deterministic coefficient (DC), the mean squared error (MSE), the mean absolute error (MAE) and the weighted mean absolute percentage error (WMAPE). According to Chen et al. [13], Tao et al. [14], Xia and Bo [15], these parameters are calculated respectively as follows:

$$
\begin{gathered}
\mathrm{DC}=1-\frac{\sum_{t=1}^{N}\left[d_{t}-D_{t}\right]^{2}}{\sum_{t=1}^{N}\left[D_{t}-\bar{D}_{o}\right]^{2}} \\
\mathrm{MSE}=\frac{1}{N} \sum_{t=1}^{N}\left(D_{t}-d_{t}\right)^{2} \\
\mathrm{MAE}=\frac{1}{N} \sum_{t=1}^{N}\left|D_{t}-d_{t}\right| \\
\text { WMAPE }=\frac{\frac{1}{N} \sum_{t=1}^{N}\left|D_{t}-d_{t}\right|}{\sum_{t=1}^{N} D_{t}} * 100
\end{gathered}
$$

where $N$ is the series length; $D_{t}$ represents the real long-term nonlinear trend or trend increment's value at time $t, d_{t}$ is the predicted long-term nonlinear trend or trend increment's value at time $t . \bar{D}_{o}$ is the mean value of the real long-term nonlinear trend or trend increment's value. Statistically, the tinier the values of the MSE, MAE and WMAPE are, the more precise the prediction accuracy of the model is. However, the closer the DC value is to 1 , the better is the model's prediction, and when $\mathrm{DC}<0.5$, the prediction is unreliable.

\section{Results and Discussion}

Figure 2 compares the trend obtains from ICEEMDAN decomposition with different $\alpha_{k}$ values for yearly high temperature among the daily minimum (YHTaDMinT) and maximum temperatures (YHTaDMaxT) time series. From Figure 2(a), it is noted that whatever the $\alpha_{k}$ values considered, the YHTaDMaxT time series trends obtained present similar pattern.

From Figure 2(b), it's observed that the YHTaDMinT time series trends obtained change slowly with $\alpha_{k}$ values. Therefore, when $\alpha_{k}$ values are chosen between 0.1 and 0.5 , the obtained trends patterns don't change significantly. Figure 3 presents the same results as in Figure 2, but for the corresponding trend increments. From these figures, it is shown that 1) the trend increments 


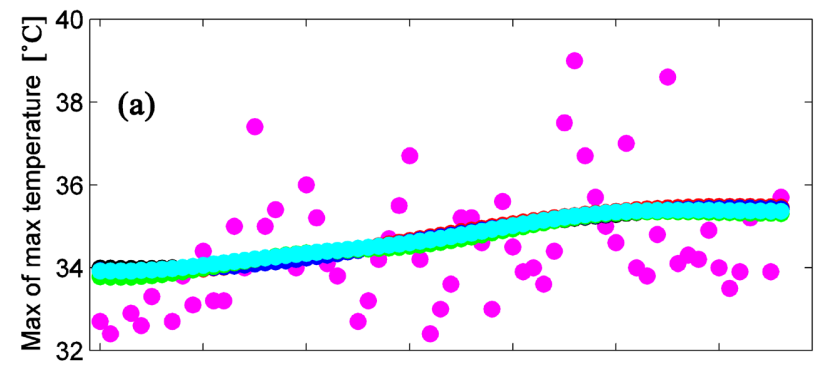
- Time series
- Trend for $\alpha_{k}=0.1$
- Trend for $\alpha_{k}=0.2$
- Trend for $\alpha_{k}=0.3$
- Trend for $\alpha_{k}=0.4$
Trend for $\alpha_{k}=0.5$

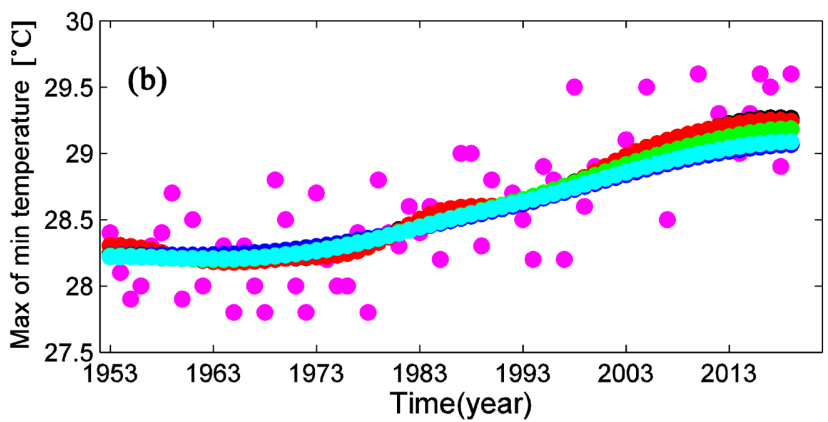

Figure 2. Trends from ICEEMDAN for YHTaDMinT and YHTaDMaxT time series. (a) For YHTaDMaxT time series; (b) for YHTaDMinT time series. Trends are shown for differents $\alpha_{k}$ values.

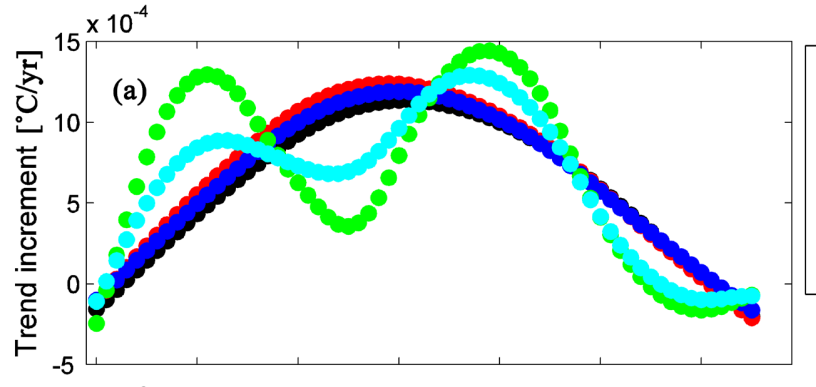

$$
\begin{aligned}
& \text { - Time series } \\
& \text { - } \text { Trend for } \alpha_{k}=0.1 \\
& \text { - } \text { Trend for } \alpha_{k}=0.2 \\
& \text { - Trend for } \alpha_{k}=0.3 \\
& \text { Trend for } \alpha_{k}=0.4
\end{aligned}
$$

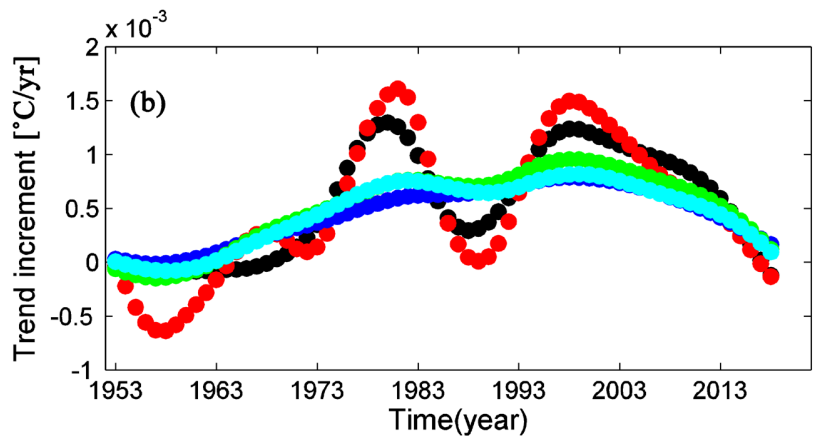

Figure 3. Same as Figure 2, but for the corresponding trend increments. (a) For YHTaDMaxT time series; (b) for YHTaDMinT time series.

don't vary monotonically like trend curves, 2) the trend increments present different forms from one $\alpha_{k}$ value to another. Thus, the trend increments patterns change significantly with $\alpha_{k}$ values.

Figure 4 compares the long-term trend obtained from ICEEMDAN for $\mathrm{YH}$ TaDMinT and YHTaDMaxT time series and the four chosen trend models (linear, quadratic, third-order, and fourth-order polynomials). The results reveal 
that YHTaDMinT and YHTaDMaxT time series are characterized by nonlinear increasing trend. Monotonically increasing trends are observed. The results have shown an increasing temperature trend in the last seventy years (1953-2019). Findings are in agreement with the global increase in temperatures indicated by IPCC [16], Trenberth et al. [17], Hartmann et al. [18], and Tang and Zhang [19]. They are also in agreement with the findings of (Trenberth et al. [17]), global temperatures are rising and will continue to do so throughout the 21 st century.

It is clearly noted that the natural trend of YHTaDMinT and YHTaDMaxT time series cannot be modeling by linear trend model, but it seems that the quadratic, third-order, and fourth-order polynomials are the good candidates to fit these trends, however, their fitting accuracy cannot be evaluated visually. Thus, to find out the functional forms of these nonlinear trends, the fitting errors of each proposed trend model are calculated and shown in Table 1.

Table 1 presents the values obtained of MSE, MAE, WMAPE and DC for the
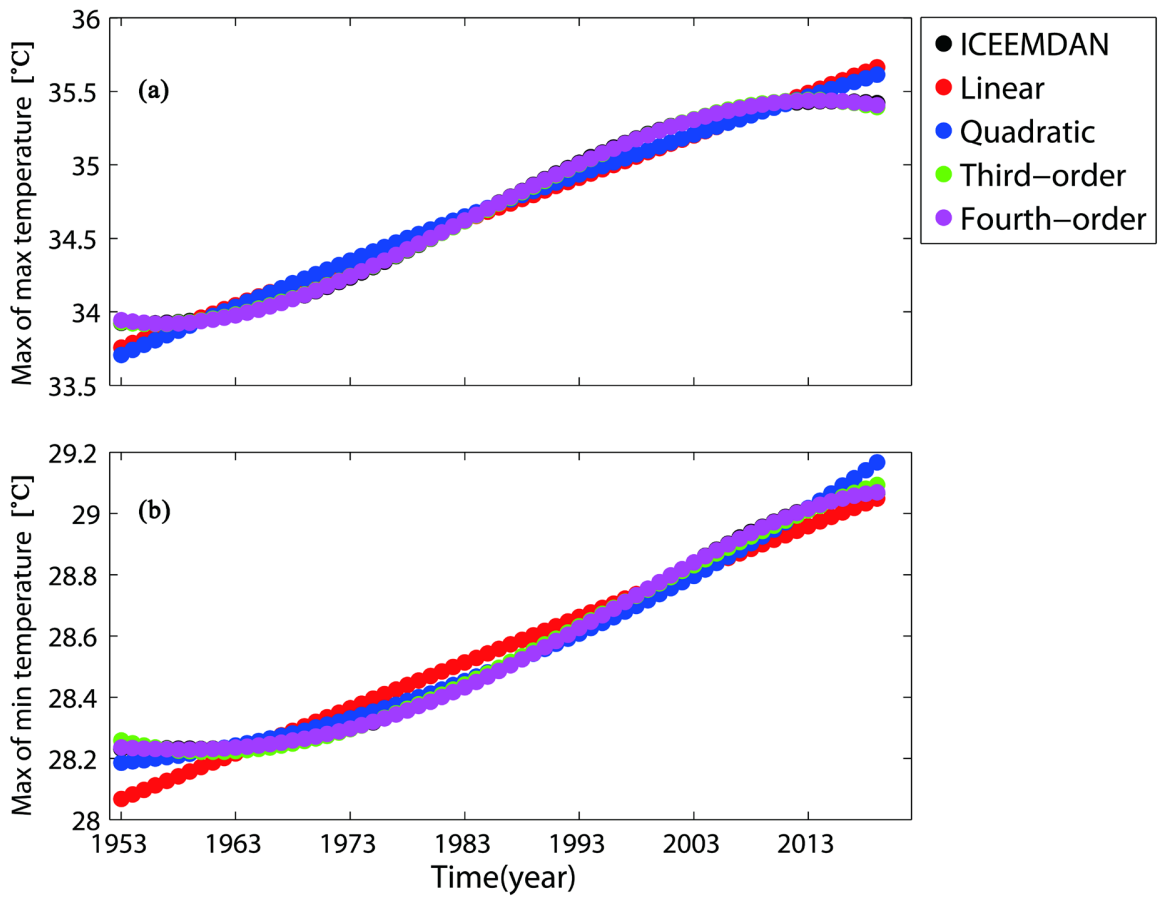

Figure 4. Trends obtained from ICEEMDAN, linear, quadratic, third-order and fourth-order polynomial fits. (a) For YHTaDMaxT time series; (b) for YHTaDMinT time series.

Table 1. Comparison of the fitting errors of each proposed models. The case of YHTaDMinT time serie are highlighted in bold.

\begin{tabular}{ccccc}
\hline Models & MSE & MAE & WMAPE (\%) & DC \\
\hline Linear & $0.0090(0.0041)$ & $0.0821(0.0550)$ & $0.0035(0.0029)$ & $0.9720(0.9530)$ \\
Quadratic & $0.0085(0.0010)$ & $0.0801(0.0265)$ & $0.0034(0.0014)$ & $0.9737(0.9884)$ \\
Third-order & $6.7 * 10^{-5}\left(8.9 * 10^{-5}\right)$ & $0.0067(0.0079)$ & $2.90 * 10^{-4}\left(4.1 * 10^{-4}\right)$ & $0.9998(0.9990)$ \\
Fourth-order & $4.2 * 10^{-5}\left(7 * 10^{-5}\right)$ & $0.0057(0.0022)$ & $2.44 * 10^{-5}\left(1.2 * 10^{-4}\right)$ & $0.9999(0.9999)$
\end{tabular}


proposed models to fit the nonlinear trend of YHTaDMinT and YHTaDMaxT time series. These values in case of YHTaDMinT time series are highlighted in bold.

It can be observed that whatever the model, the time series (YHTaDMinT or YHTaDMaxT) considered the MSE, MAE, WMAPE values (Table 1) of the third-order polynomial and fourth-order polynomial are significantly smaller than those of quadratic polynomial function. Therefore, the third-order and fourth-order polynomial are both the good candidates to modelize the functional form of the real long-term nonlinear trend of YHTaDMinT and YHTaDMaxT time series at Cotonou city. Moreover, the DC values obtained for both functions are closer to 1 , therefore, the better are their fitting. However, the DC values of the fourth-order polynomial are greater than those of the third-order polynomial. Thus, fourth-order polynomial function is optimal among the four trend models proposed in this study.

Figure 5 compares the long-term trend increment obtained from ICEEMDAN for YHTaDMinT and YHTaDMaxT time series and four trend increment models (linear, quadratic, third-order, and fourth-order polynomials). The real trend increment obtained from ICEEMDAN for YHTaDMaxT time series (Figure 5(a), curve in black) increases from 1953 to 1983 and decreases from 1984 to 2019. However, for the YHTaDMinT time series, it increases from 1963 to 1983 and decreases from 1993 to 2019. For the both time series, the trend increments are not the monotonic functions. It is clearly noted that the natural trend increments of YHTaDMinT and YHTaDMaxT time series cannot be modeling by linear
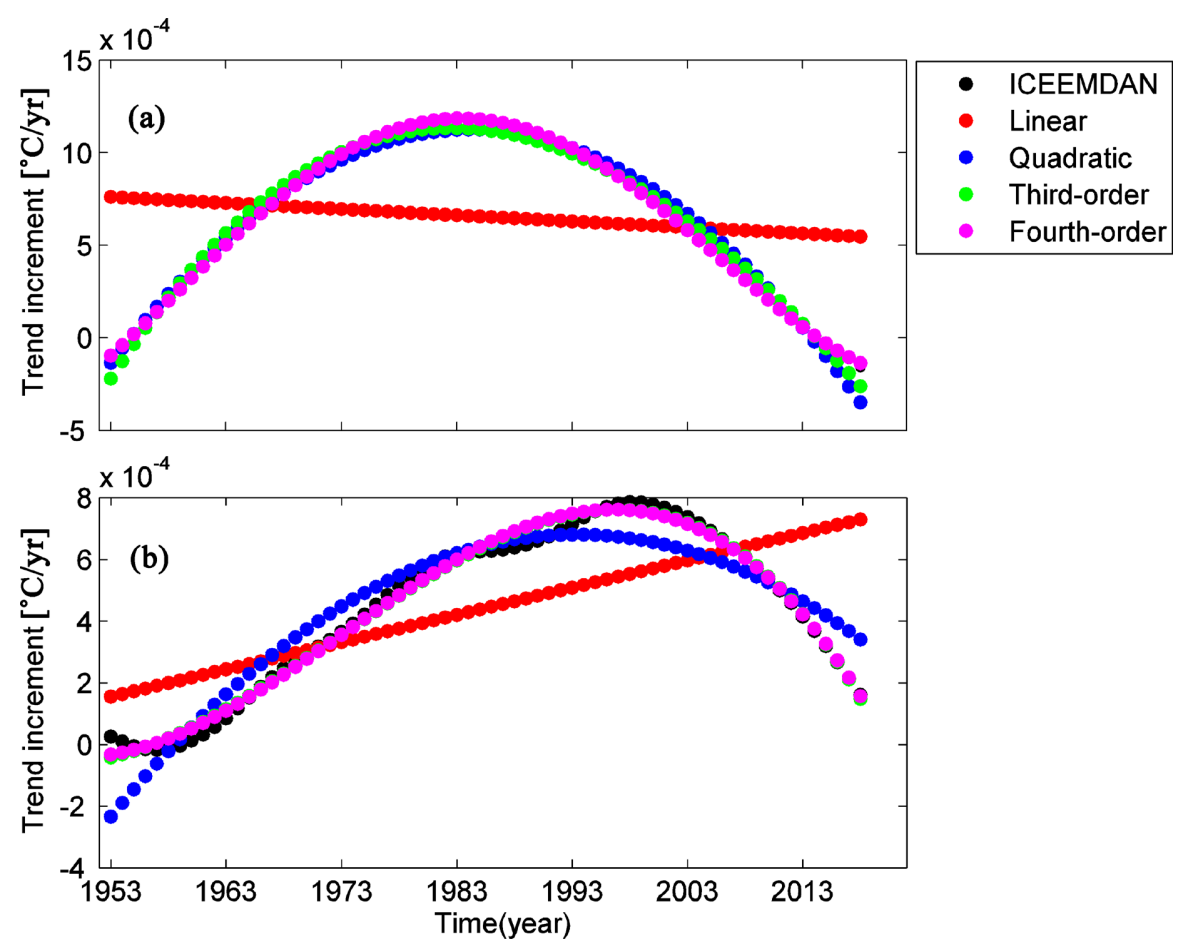

Figure 5. Same as in Figure 4 but for trend increment. (a) For YHTaDMaxT time series; (b) for YHTaDMinT time series. 
Table 2. Same as Table 1, but for the trend increment.

\begin{tabular}{ccccc}
\hline Models & MSE & MAE & WMAPE (\%) & DC \\
\hline Linear & $1.8^{*} 10^{-7}\left(4.02 * 10^{-8}\right)$ & $3.7 * 10^{-4}\left(1.7 * 10^{-4}\right)$ & $0.8641(0.5831)$ & $0.0214(0.4136)$ \\
Quadratic & $3.7 * 10^{-9}\left(7.1 * 10^{-9}\right)$ & $5.1 * 10^{-5}\left(6.74 * 10^{-5}\right)$ & $0.1174(0.2313)$ & $0.9798(0.8965)$ \\
Third-order & $2.4 * 10^{-9}\left(7.33 * 10^{-10}\right)$ & $4.3 * 10^{-5}\left(2.20 * 10^{-5}\right)$ & $0.0989(0.0755)$ & $0.9868(0.9893)$ \\
Fourth-order & $6.4 * 10^{-11}\left(7.21 * 10^{-10}\right)$ & $6.7 * 10^{-6}\left(2.18^{*} 10^{-5}\right)$ & $0.0156(0.0749)$ & $0.9997(0.9895)$ \\
\hline
\end{tabular}

trend model, but it seems that the quadratic, third-order, and fourth-order polynomials are good candidates for YHTaDMaxT time series. Thus, to find out the functional form of these trend increments, the fitting errors of each proposed trend increments model are calculated and shown in Table 2.

Table 2 presents the MSE, MAE, WMAPE and DC values for the proposed models to fit the nonlinear trend increment of YHTaDMinT and YHTaDMaxT time series. The values of the MSE, MAE, WMAPE and DC values in case of YHTaDMinT time serie are highlighted in bold. It can be observed that whatever the model, the time series (YHTaDMinT or YHTaDMaxT) considered, the MSE, MAE, WMAPE values (Table 2) of the third-order polynomial and fourth-order polynomial are significantly smaller than those of quadratic polynomial function. Therefore, the third-order and fourth-order polynomial are the good candidates to modelize the functional form of the real long-term nonlinear trend increment of YHTaDMinT and YHTaDMaxT time series at Cotonou city. The DC values of the fourth-order polynomial function are closer to 1 and greater than those of the third-order polynomial. Thus, fourth-order polynomial function is optimal among the four trend increment models.

\section{Summary and Conclusions}

In this paper, the real long-term nonlinear trend and the changing rate of the yearly high temperature among the daily minimum (YHTaDMinT) and maximum temperatures (YHaDMaxT) observed from 1953 to 2019 at Cotonou city, the most industrialized and developed city in Benin Republic are estimated by ICEEMDAN method and, the best polynomial trend model among four trend models and trend increment of these nonlinear trends and trend increment is found out. The major remarks are summarized as follows: 1) YHTaDMinT and YHTaDMaxT time series are characterized by nonlinear and monotonically increasing trends. The trend increments don't vary monotonically; they present different phases in their variation. 2) Based on four indicators, such as the deterministic coefficient (DC), mean squared error (MSE), mean absolute error (MAE) and weighted mean absolute percentage error (WMAPE), for both time series, the fourth-order polynomial is optimal among the four chosen trend models (linear, quadratic, third-order and fourth-order polynomial function). For the trend increment, the fourth-order polynomial function presents a good performance in terms of trend increments estimation. This study reveals the 
evidence of climate change in Cotonou, Benin republic. The findings of the present research can be used in climate change impacts studies and in assessing environmental heat stress on the health of the population in Cotonou city. Future works will be focused on the investigation of the long-term trends in the climatic variables simulated by regional climate models in Benin republic and its best functional forms.

\section{Data Availability}

The data used in this study are supplied by the local service of Météo-Bénin in Cotonou. The data are not available online in any data base, so that we cannot provide a link to reach them. They are provided when researchers address requests to Météo-Bénin (http://www.asecna.aero).

\section{Acknowledgements}

The authors would like to thank METEO-Benin for providing the data used to conduct the study. We also express their great appreciation to the editor and the anonymous referees for their valuable suggestions.

\section{Funding}

This study received no specific funds.

\section{Conflicts of Interest}

The authors declare no conflicts of interest regarding the publication of this paper.

\section{References}

[1] Adarsh, S. and Janga Reddy, M. (2017) Investigating the Multiscale Variability and Teleconnections of Extreme Temperature over Southern India Using the Hilbert-Huang Transform. Modeling Earth Systems and Environment, 3, Article No. 8. https://doi.org/10.1007/s40808-017-0281-4

[2] Djanna Koubodana, H., Adounkpe, J., Tall, M., Amoussou, E., Atchonouglo, K. and Mumtaz, M. (2020) Trend Analysis of Hydro-Climatic Historical Data and Future Scenarios of Climate Extreme Indices over Mono River Basin in West Africa. American Journal of Rural Development, 8, 37-52. https://doi.org/10.20944/preprints201906.0267.v1

[3] Jiang, L., Zhao, L. and Zhao, Z. (2017) On the Difference of Scaling Properties for Temperature and Precipitation over China. Advances in Meteorology, 2017, Article ID: 5761275. https://doi.org/10.1155/2017/5761275

[4] Luo, M., Leung, Y., Zhou, Y. and Zhang, W. (2015) Scaling Behaviors of Global Sea Surface Temperature. Journal of Climate, 28, 3122-3132. https://doi.org/10.1175/JCLI-D-13-00743.1

[5] Solomon, S., Qin, D., Manning, M., Chen, Z., Marquis, M. and Averyt, K.B. (2007) Technical Summary. In: Climate Change 2007: 2e Physical Science Basis, Contribution of Working Group I to the Fourth Assessment Report of the Intergovernmental Panel on Climate Change, Cambridge University Press, Cambridge, 19-92. 
[6] Abaje, I.B. and Oladipo, E.O. (2019) Recent Changes in the Temperature and Rainfall Conditions over Kaduna State, Nigeria. Ghana Journal of Geography, 11, 127-157.

[7] Gadedjisso-Tossou, A., Adjegan, K.I. and Kablan, A.K.M. (2021) Rainfall and Temperature Trend Analysis by Mann-Kendall Test and Significance for Rainfed Cereal Yields in Northern Togo. Science, 3, Article No. 17. https://doi.org/10.3390/sci3010017

[8] Agbazo, M.N., Koto N’Gobi, G., Alamou, E., Kounouhewa, B. and Afouda, A. (2019) Fractal Analysis of the Long-Term Memory in Precipitation over Bénin Republic (West Africa). Advances in Meteorology, 2019, Article ID: 1353195. https://doi.org/10.1155/2019/1353195

[9] Huang, N.E., Shen, Z., Long, S.R. M., Wu, Z., Shih, E.H., Zheng, Q., Tung, C.C. and Liu, H.H. (1998) The Empirical Mode Decomposition and the Hilbert Spectrum for Nonlinear and Nonstationary Time Series Analysis. Proceedings of the Royal Society of London A, 454, 903-993. https://doi.org/10.1098/rspa.1998.0193

[10] Wu, Z. and Huang, N.E. (2009) Ensemble Empirical Mode Decomposition: A Noise-Assisted Data Analysis Method. Advances in Adaptive Data Analysis, 1, 1-41. https://doi.org/10.1142/S1793536909000047

[11] Torres, M.E., Colominas, M.A., Schlotthauer, G. and Flandrin, P. (2011) A Complete Ensemble Empirical Mode Decomposition with Adaptive Noise. IEEE International Conference on Acoustics, Speech and Signal Processing, 125, 4144-4147. https://doi.org/10.1109/ICASSP.2011.5947265

[12] Colominas, M.A., Schlotthauer, G. and Torres, M.E. (2014) Improved Complete Ensemble EMD: A Suitable Tool for Biomedical Signal Processing. Biomedical Signal Processing and Control, 14, 19-29. https://doi.org/10.1016/j.bspc.2014.06.009

[13] Chen, Y., Li, P., Ren, W., Shen, X. and Cao, M. (2020) Field Data-Driven Online Prediction Model for Icing Load on Power Transmission Lines. Measurement and Control, 53, 126-140. https://doi.org/10.1177/0020294019878872

[14] Tao, W., Chen, J., Gui, Y. and Kong, P. (2020) Coking Energy Consumption Radial Basis Function Prediction Model Improved by Differential Evolution Algorithm. Measurement and Control, 52, 1122-1130. https://doi.org/10.1177/0020294019858182

[15] Xia, B.K. and Bo, Y.C. (2010) A Soft Sensor for Water-Content-in-Oil Based on GA-RBF Neural Network. Measurement and Control, 43, 179-182. https://doi.org/10.1177/002029401004300604

[16] IPCC (2007) Climate Change 2007: Synthesis Report. In: Pachauri, R.K. and Reisinger, A., Eds., Contribution of Working Groups I, II and III to the Fourth Assessment Report of the Intergovernmental Panel on Climate Change, Intergovernmental Panel on Climate Change, Geneva, 104 p.

[17] Trenberth, K.E., Jones, P.D., Ambenje, P., Bojariu, R., Easterling, D., Klein Tank, A., Zhai, P., et al. (2007) Observations: Surface and Atmospheric Climate Change. In: Solomon, S., Qin, D., Manning, M., Chen, Z., Marquis, M., Averyt, K.B., Miller, H.L., et al., Eds., Climate Change 2007: The Physical Science Basis, Contribution of Working Group I to the Fourth Assessment Report of the Intergovernmental Panel on Climate Change (IPCC), Cambridge University Press, New York, 235-336.

[18] Hartmann, D.L., Klein Tank, A.M.G., Rusticucci, M., Alexander, L.V., Brönnimann, S., Charabi, Y., Zhai, P.M., et al. (2013) Observations: Atmosphere and Surface. In Stocker, T.F., Qin, D., Plattner, G.-K., Tignor, M., Allen, S.K., Boschung, J., Midgley, P.M., et al., Eds., Climate Change 2013: The Physical Science Basis, Contribution of Working Group I to the Fifth Assessment Report of the IPCC, Cambridge 
University Press, Cambridge, 159-254.

[19] Tang, L. and Zhang, Y. (2018) Considering Abrupt Change in Rainfall for Flood Season Division: A Case Study of the Zhangjia Zhuang Reservoir, Based on a New Model. Water, 10, Article No. 1152. https://doi.org/10.3390/w10091152 